Review Article

\title{
Genetic Association between Methylenetetrahydrofolate Reductase Gene Polymorphism and Risk of Osteonecrosis of the Femoral Head
}

\author{
Wei Chai, ${ }^{1}$ Zhendong Zhang, ${ }^{1}$ Ming Ni, ${ }^{1}$ Peiliang Geng, ${ }^{2}$ Zijian Lian, ${ }^{3}$ Guoqiang Zhang, \\ Lewis L. Shi, ${ }^{4}$ and Jiying Chen ${ }^{1}$ \\ ${ }^{1}$ Department of Orthopaedics, Chinese People's Liberation Army General Hospital, 301 Orthopaedic Hospital, 28 Fuxing Road, \\ Haidian District, Beijing 100853, China \\ ${ }^{2}$ Cancer Center, Division of Internal Medicine, Chinese PLA General Hospital \& Chinese PLA Medical School, 28 Fuxing Road, \\ Haidian District, Beijing 100853, China \\ ${ }^{3}$ Medical School of Nankai University, 94 Weijin Road, Tianjin 300071, China \\ ${ }^{4}$ Department of Orthopaedics, University of Chicago Hospital, 5841 S. Maryland Avenue, Chicago, IL 60637, USA
}

Correspondence should be addressed to Jiying Chen; chenjiying_301@163.com

Received 3 July 2014; Accepted 24 September 2014

Academic Editor: Dongquan Shi

Copyright (c) 2015 Wei Chai et al. This is an open access article distributed under the Creative Commons Attribution License, which permits unrestricted use, distribution, and reproduction in any medium, provided the original work is properly cited.

\begin{abstract}
Background. Methylenetetrahydrofolate reductase (MTHFR) SNP rs1801133 has been frequently investigated in recent years. Relevant candidate gene association studies with this SNP and osteonecrosis of the femoral head (ONFH) reported conflicting results. Meta-analysis provides a method to combine these data and to determine the association in a larger sample size. Method. We conducted a systematic search to identify possible studies. Four pooled ORs (odds ratios, T versus C, TT versus CC, TT/CT versus CC, and TT versus CT/CC), along with $95 \%$ confidence interval (CI), were calculated to evaluate the association between SNP rs1801133 and ONFH susceptibility. Both fixed effects model and random effects model were used. Findings. We eventually included twelve studies in this analysis, with results showing no overall association between ONFH susceptibility and SNP rs1801133 ( $\mathrm{T}$ versus $\mathrm{C}: \mathrm{OR}=1.15,95 \% \mathrm{CI}=0.97-1.38$; $\mathrm{TT}$ versus $\mathrm{CC}: \mathrm{OR}=1.15,95 \% \mathrm{CI}=0.91-1.46$; $\mathrm{TT} / \mathrm{CT}$ versus $\mathrm{CC}$ : $\mathrm{OR}=1.09,95 \%$ $\mathrm{CI}=0.95-1.25$; and TT versus CT/CC: $\mathrm{OR}=1.16,95 \% \mathrm{CI}=0.93-1.45)$. When stratified based on ethnicity, the results were still not significant. Conclusion. Our findings are generally supportive of no association between MTHFR SNP rs1801133 and the etiology of ONFH.
\end{abstract}

\section{Introduction}

Osteonecrosis of the femoral head (ONFH) is a painful disorder of the hip and features necrosis of femoral subchondral bone [1]. This disease is often observed in young adults and is frequently progressive. ONFH will often evolve into subchondral bone fracture, femoral head collapse, and subsequent hip joint degeneration, leading to poor long term outcomes [2]. The collapse of femoral head often leads to loss of function [3-5]. The pathophysiology of ONFH remains incompletely elucidated; intravascular coagulation may represent a pathogenetic mechanism resulting in bone ischemia and death [6]. Coagulation abnormalities in patients with hip osteonecrosis are associated with an increase in risk of developing bone necrosis by predisposing thromboembolic phenomena [7].

A large number of outstanding researchers have identified multiple factors that genetically contribute to hypercoagulability and osteonecrosis, including protein $\mathrm{C}$ and protein $\mathrm{S}$ deficiency, factor V Leiden, prothrombin G20210A mutation, and higher lipoprotein levels [8-10]. In recent studies, several gene single nucleotide polymorphisms (SNPs) associated with thrombophilia have been implicated in ONFH [11]. Methylenetetrahydrofolate reductase (MTHFR) is an enzyme which plays a key role in the remethylation of homocysteine. A $\mathrm{C} \rightarrow \mathrm{T}$ mutation at nucleotide 677 leading to a valine 
substitution for an alanine $(677 \mathrm{C}>\mathrm{T}, \mathrm{rs1801133})$ is reported to be functionally relevant to MTHFR enzymatic activation, homocysteine levels, and thrombotic events $[12,13]$.

Extensive research into the etiology of ONFH to date has focused on SNP rs1801133. Results of a study by Glueck et al. in 2001 showed that SNP rs1801133 was a genetic risk factor predisposing susceptibility to ONFH [14]. Recently, Li et al. investigated the association of the SNP with risk of ONFH in a sample of Chinese patients, finding supportive evidence of a significant association as well [15]. However, most replication studies reported that SNP rs1801133 is not a risk factor for the bone disease [16-18]. Hence, we hypothesize that the ONFH susceptibility risks may not be attributable to SNP rs1801133. In an effort to test this hypothesis, we performed a metaanalysis to combine all published data and to determine the association in a larger sample size.

\section{Methods}

2.1. Study Search and Selection. A systematic search of the PubMed (http://www.ncbi.nlm.nih.gov/sites/entrez/) and CNKI (http://www.cnki.net/) electronic database was conducted up to October 1, 2013, to identify all studies investigating the association between SNP rs1801133 and ONFH risk. "Osteonecrosis of the femoral head," "ONFH," "Methylenetetrahydrofolate reductase," "MTHFR," and "polymorphism" were the keywords we used in the search. The electronic search was complemented with a manual search of citations of all original articles.

There were three inclusion criteria for the meta-analysis. First, the study must investigate the association between SNP rs1801133 and ONFH risk by comparing ONFH patients with healthy or ONFH-free control subjects. Second, the study had to provide detailed genotype frequency or contain sufficient data with which we could calculate the frequencies of TT, CT, and TT. Third, the subjects must be humans and cannot be repeatedly used; if they were, we included the study with the largest number of subjects.

2.2. Data Extraction. Using the inclusion criteria listed above, two investigators picked out all eligible studies and extracted the following data from each of them: authors, year of publication, country of study, ethnicity, genotyping method, genotypic frequencies, and type of controls. Disagreements were resolved by discussion.

2.3. Statistical Analysis. We first checked the deviation from Hardy-Weinberg equilibrium (HWE) in control subjects using a chi-squared goodness-of-fit test. To evaluate the strength of the genetic association, four pooled ORs (odds ratios, T versus $\mathrm{C}$, TT versus $\mathrm{CC}$, TT/CT versus $\mathrm{CC}$, and TT versus CT/CC) were calculated, along with the $95 \%$ confidence intervals (CIs). A statistical test described by Cochran was conducted to measure heterogeneity between studies [19]. In case of absence of heterogeneity, the pooled ORs were calculated assuming a fixed effects model [20], or a random effects model was used [21]. To identify if included studies influenced summary estimates substantially, we performed sensitivity analysis by omitting one study at a time. Publication bias was qualitatively assessed using the funnel plots and Egger's test [22]. All statistical tests were performed with the software Stata version 12.0 (Stata Corporation, College station, TX, USA). $P$ value $<0.05$ was considered as significant.

\section{Results}

3.1. Extraction Process and Study Characteristics. Figure 1 summarizes the process of study identification. We yielded a total of 331 relevant articles (PubMed, 207; CNKI, 124). Evaluation of title, abstract, and full-text led to 12 eligible studies [14-18, 23-29]. General characteristics of the included studies are summarized in Table 1 . Seven studies investigated the association of SNP rs1801133 with ONFH susceptibility in a sample of Caucasian population and five in Asian populations. Seven studies used healthy control subjects and four used ONFH-free subjects, with one not reporting such information. Most studies used polymerase chain reactionrestriction fragment length polymorphism (PCR-RFLP) to genotype SNP rs1801133. Deviation from HWE was tested in three studies $[15,16,26]$.

3.2. Meta-Analysis Results. As shown in Table 2, all of the genetic models we tested showed that individuals with $\mathrm{T}$ allele appeared to be at higher risk of ONFH ( $\mathrm{T}$ versus $\mathrm{C}$ : OR = $1.15,95 \% \mathrm{CI}=0.97-1.38$; $\mathrm{TT}$ versus CC: $\mathrm{OR}=1.15,95 \% \mathrm{CI}$ $=0.91-1.46$; TT/CT versus CC: $\mathrm{OR}=1.09,95 \% \mathrm{CI}=0.95-$ 1.25; and TT versus CT/CC: $\mathrm{OR}=1.16,95 \% \mathrm{CI}=0.93-1.45$ ) (Figure 2), although this was not statistically significant. In terms of stratified analysis by ethnicity, the results for both Caucasian and Asian populations were similar to those for the overall population (Table 2 and Figure 2).

3.3. Heterogeneity Test and Sensitivity Analyses. We observed no significant heterogeneity for SNP rs1801133 under all genetic models with the exception of $\mathrm{T}$ versus C $\left(P_{\text {Heterogeneity }}=0.027\right) \quad($ Table 2$)$. Sensitivity analyses were conducted, and it was identified that $\mathrm{Li}$ et al. [15] and Kutlar et al. [16] were the main sources of heterogeneity, exclusion of which effectively increased homogeneity without significantly influencing the estimates of overall ONFH risk $\left(P_{\text {Heterogeneity }}=\right.$ $0.223, \mathrm{OR}=1.03,95 \% \mathrm{CI}=0.92-1.17)$. This outcome assured the stability and reliability of our results.

3.4. Publication Bias. We evaluated the publication bias using the tests described by Begg and Egger. The shape of all funnel plots was symmetrical $(P>0.05)$. Begg's test also showed that there was little publication bias across studies $(P>0.05)$ (Figure 3 for TT/CT versus CC).

\section{Discussion}

SNPs represent the most common type of DNA variation in human. Genetic association studies are being widely and appropriately used to identify potential genetic prognostic 


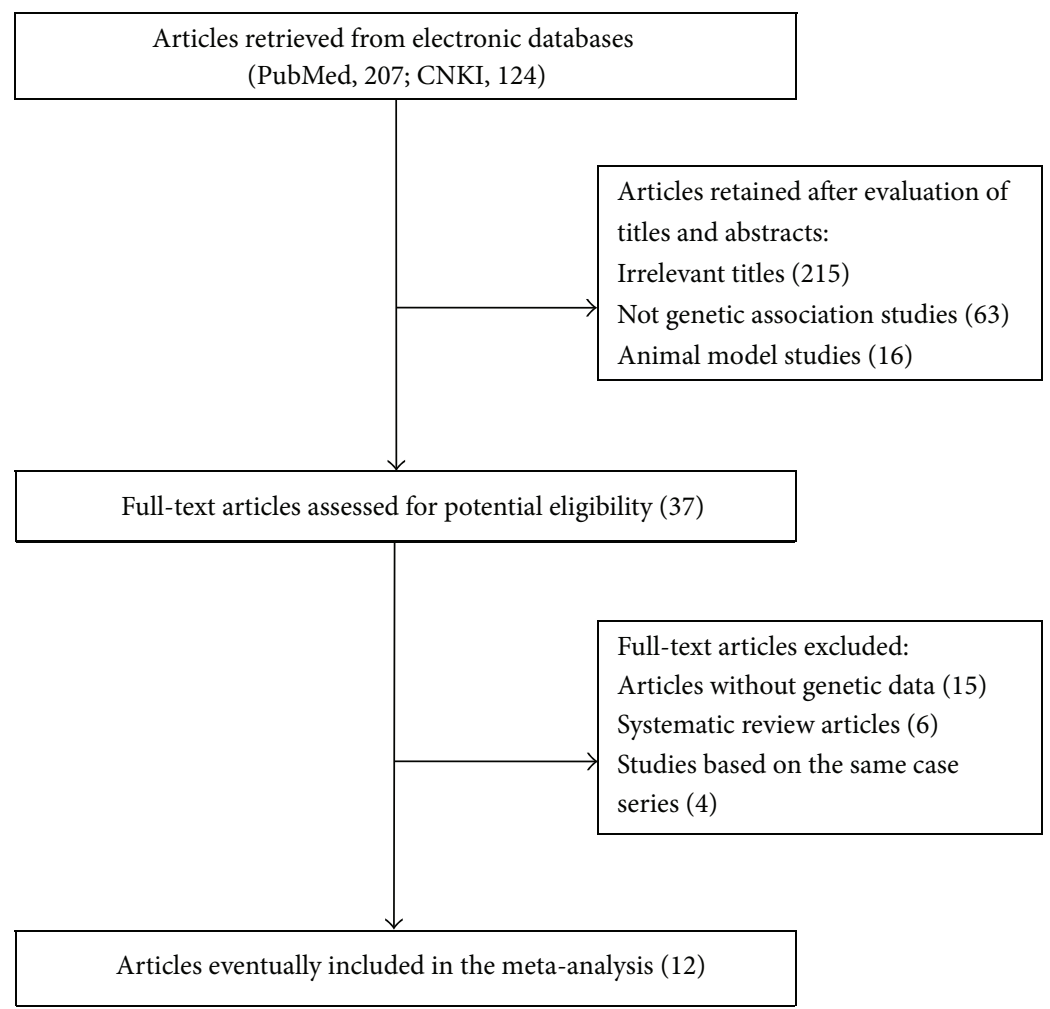

FIGURE 1: Flow chart of study identification and specific reasons for exclusion from the meta-analysis.

TABLE 1: General characteristics of the studies included in the present meta-analysis.

\begin{tabular}{|c|c|c|c|c|c|c|c|}
\hline \multirow{2}{*}{ ID } & \multirow{2}{*}{ Study } & \multirow{2}{*}{ Publication year } & \multirow{2}{*}{ Ethnicity (country) } & \multirow{2}{*}{ Control source } & \multirow{2}{*}{ Genotyping methods } & \multicolumn{2}{|c|}{ Sample size } \\
\hline & & & & & & Case & Control \\
\hline 1 & Glueck et al. [23] & 1999 & Caucasian (USA) & Healthy subjects & PCR-RFLP & 59 & 234 \\
\hline 2 & Glueck et al. [14] & 2001 & Caucasian (USA) & Healthy subjects & PCR-RFLP & 36 & 235 \\
\hline 3 & Kutlar et al. [16] & 2001 & Caucasian (USA) & ONFH-free subjects & PCR-RFLP & 45 & 62 \\
\hline 4 & Zalavras et al. [24] & 2002 & Caucasian (Greece) & Healthy subjects & PCR-RFLP & 66 & 300 \\
\hline 5 & Bernbeck et al. [17] & 2003 & Caucasian (Germany) & NS & NS & 14 & 73 \\
\hline 6 & Asano et al. [18] & 2004 & Asian (Japan) & ONFH-free subjects & DS & 31 & 106 \\
\hline 7 & Celik et al. [25] & 2006 & Caucasian (Turkey) & ONFH-free subjects & PCR-RFLP & 11 & 39 \\
\hline 8 & Chang et al. [26] & 2008 & Asian (Korea) & Healthy subjects & Multiplex PCR & 71 & 200 \\
\hline 9 & Liu et al. [27] & 2009 & Asian (China) & Healthy subjects & PCR-RFLP & 89 & 77 \\
\hline 10 & Kim et al. [28] & 2010 & Asian (Korea) & ONFH-free subjects & TaqMan & 438 & 269 \\
\hline 11 & Gagala et al. [29] & 2013 & Caucasian (Poland) & Healthy subjects & PCR-RFLP & 68 & 100 \\
\hline 12 & Li et al. [15] & 2013 & Asian (China) & Healthy subjects & PCR-RFLP & 93 & 83 \\
\hline
\end{tabular}

PCR-RFLP-polymerase chain reaction-restriction fragment length polymorphism, NS-data not shown, DS-direct sequencing.

factors and molecular biomarkers for genetically determined human diseases [30]. Due to their impact on the biological function of corresponding genes, candidate gene association studies have given priority to the well-characterized SNPs such as rs1801133 in exon 4 of the MTHFR gene. Kim et al. conducted a comparison of ONFH and control subjects using logistic regression models, revealing no significant association between SNP rs1801133 and ONFH susceptibility in a Korean population [28]. Chang et al., however, showed evidence that supported the involvement of this SNP in development of ONFH [26]. The disparate results across the studies may at least partly be attributable to the relatively small sample sizes. This led us to perform a meta-analysis to determine the association in a larger sample size.

When pooling all published data together, we found little evidence of a significant association between SNP rs1801133 and ONFH susceptibility, consistent with our hypothesis. A review of overall progress and contribution of candidate gene association studies to current understanding of the genetic susceptibility to cancer suggested that MTHFR C677T increased risk of gastric cancer (OR, 1.52; 95\% CI, 1.31-1.77) [31]. Variation of SNP rs1801133 is associated with 
TABLE 2: Meta-analysis of the association between MTHFR SNP rs1801133 and ONFH risk.

\begin{tabular}{|c|c|c|c|c|c|}
\hline \multirow{2}{*}{ Contrast model } & \multirow{2}{*}{ Studies } & \multicolumn{2}{|c|}{ Odds ratio } & \multirow{2}{*}{ Model } & \multirow{2}{*}{$P_{\text {Heterogeneity }}$} \\
\hline & & OR $(95 \% \mathrm{CI})$ & $P_{\mathrm{OR}}$ & & \\
\hline Total studies & 12 & & & & \\
\hline$T$ versus $C$ & 11 & $1.15(0.97,1.38)$ & 0.095 & Random & 0.027 \\
\hline TT versus CC & 11 & $1.15(0.91,1.46)$ & 0.227 & Fixed & 0.234 \\
\hline TT/CT versus CC & 12 & $1.09(0.95,1.25)$ & 0.222 & Fixed & 0.245 \\
\hline TT versus CT/CC & 11 & $1.16(0.93,1.45)$ & 0.178 & Fixed & 0.188 \\
\hline Caucasians & 7 & & & & \\
\hline T versus $\mathrm{C}$ & 6 & $1.12(0.82,1.53)$ & 0.428 & Random & 0.073 \\
\hline TT versus CC & 6 & $0.99(0.62,1.61)$ & 0.983 & Fixed & 0.336 \\
\hline TT/CT versus CC & 7 & $1.13(0.90,1.41)$ & 0.295 & Fixed & 0.208 \\
\hline TT versus CT/CC & 6 & $0.93(0.59,1.46)$ & 0.742 & Fixed & 0.258 \\
\hline Asians & 5 & & & & \\
\hline T versus $\mathrm{C}$ & 5 & $1.19(0.93,1.51)$ & 0.138 & Random & 0.037 \\
\hline TT versus CC & 5 & $1.21(0.92,1.58)$ & 0.164 & Fixed & 0.141 \\
\hline TT/CT versus CC & 5 & $1.07(0.90,1.27)$ & 0.460 & Fixed & 0.271 \\
\hline TT versus CT/CC & 5 & $1.25(0.97,1.60)$ & 0.086 & Fixed & 0.161 \\
\hline
\end{tabular}

OR: odds ratio, 95\% CI: 95\% confidence interval, Random: random effects model, Fixed: fixed effects model.

\begin{tabular}{|c|c|c|}
\hline Study ID & OR (95\% CI) & Weight (\%) \\
\hline \multicolumn{3}{|l|}{ Caucasian } \\
\hline Glueck (1999) & $0.79(0.49,1.29)$ & 9.41 \\
\hline Glueck (2001) & $1.48(0.85,2.56)$ & 5.05 \\
\hline Kutlar (2001) & $2.76(1.09,6.99)$ & 1.38 \\
\hline Zalavras (2002) & $1.09(0.72,1.67)$ & 10.24 \\
\hline Celik (2006) & $0.25(0.03,2.14)$ & 1.19 \\
\hline Gagala (2013) & $1.17(0.69,1.97)$ & 6.54 \\
\hline Bernbeck (2003) & $1.24(0.51,3.04)$ & 2.13 \\
\hline Subtotal $\left(I^{2}=28.9 \%, P=0.208\right)$ & $1.13(0.90,1.41)$ & 35.94 \\
\hline \multicolumn{3}{|l|}{. } \\
\hline \multicolumn{3}{|l|}{ Asian } \\
\hline Asano (2004) & $0.87(0.45,1.69)$ & 4.72 \\
\hline Chang (2008) & $1.24(0.82,1.90)$ & 9.65 \\
\hline Liu (2009) & $1.11(0.71,1.76)$ & 8.77 \\
\hline Kim (2010) & $0.93(0.73,1.18)$ & 34.66 \\
\hline $\mathrm{Li}(2013)$ & $1.64(1.00,2.69)$ & 6.26 \\
\hline Subtotal $\left(I^{2}=22.5 \%, P=0.271\right)$ & $1.07(0.90,1.27)$ & 64.06 \\
\hline . & & \\
\hline Overall $\left(I^{2}=20.2 \%, P=0.245\right)$ & $1.09(0.95,1.25)$ & 100.00 \\
\hline $\begin{array}{c}1 \\
0.0299\end{array}$ & $\begin{array}{c}1 \\
33.4\end{array}$ & \\
\hline
\end{tabular}

FIGURE 2: Meta-analysis using a fixed effects model for the association between MTHFR SNP rs1801133 and ONFH susceptibility (TT/CT versus $\mathrm{CC}$ ) is illustrated in analysis stratified by ethnicity. OR: odds ratio; CI: confidence interval. 


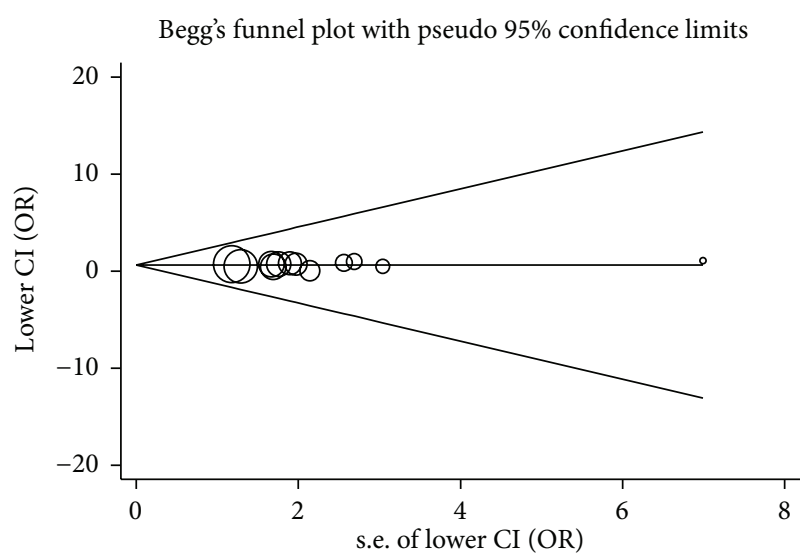

Figure 3: Funnel plot of publication bias for TT/CT versus CC. Begg's test $P=0.653$; the circles represent the weight of each study.

decreased enzymatic activity and elevated homocysteine levels. Increased frequency of this SNP has been detected among patients with cardiovascular disease and coronary atherosclerotic disease $[12,32]$. These data are suggestive of a possible effect on human diseases attributed to SNP rs1801133. On the other hand, we cannot exclude the possibility that these diseases are etiologically different and it is likely that SNP rs1801133 may not represent a contributing factor for ONFH. However, this aspect is worth to be proven with a very large-sized study.

Further analysis stratified by ethnicity also showed no association in Asians and in Caucasians. It has previously been reported that CT and TT genotypes have 65\% and 30\% of the MTHFR activity, respectively, suggesting individuals with these genotypes are at higher risk of various diseases, such as ONFH [33]. A frequency ranging from 5\% to $40 \%$ in European populations [32] implicated that SNP rs1801133 may contribute to ONFH, especially among Caucasians. But the expected association was not detected in our study, which may still be caused by the limited sample size.

Results of a previous meta-analysis showed an association between MTHFR SNP rs1801133 and ONFH in non-Asian populations [34]. In this analysis, a total of eight studies (five on Caucasians) with 778 cases and 1,162 controls were included, possibly leading to a less reliable and incomprehensive conclusion because of the smaller sample. We expanded our analysis by adding additional four studies, with no significant association identified. The uncertainty highlights the necessity to conduct subsequent studies to validate the presently unclear genetic association.

There are some limitations to this study. The ONFH individuals in our study were caused by different etiologies, including primary ONFH without special history, glucocorticoid intake, and alcohol abuse, which may affect the final results. There were significant heterogeneity among the studies, including ethnicity, number of individuals, and methodology. The total sample size may not generate sufficient power to detect the overall and ethnicity-specific effects in subgroups, and minor or moderate association may be masked. Additionally, the nonstandardized selection of control population, such as healthy versus ONFH-free subjects, may introduce nondifferential misclassification bias. Lastly, estimation of age in ONFH susceptibility was not conducted due to lack of original data.

In conclusion, the current meta-analysis revealed that MTHFR SNP rs1801133 was not significantly associated with the development of ONFH. We found similar results in ethnicity-specific analysis. Considering ONFH can be caused by many factors such as application of glucocorticoid and alcoholism, these findings need further validation through future larger studies stratified by different etiological factors.

\section{Conflict of Interests}

The authors have declared that no conflict of interests exists.

\section{Authors' Contribution}

Wei Chai, Ming Ni, and Jiying Chen conceived and designed the research. Wei Chai, Peiliang Geng, and Guoqiang Zhang performed the research. Analysis and interpretation of data were done by Lewis L. Shi, Wei Chai, Ming Ni, Guoqiang Zhang, Zijian Lian, and Zhendong Zhang. Drafting and revising then writing the paper were done by Wei Chai, Zhendong Zhang, and Lewis L. Shi. Wei Chai and Zhendong Zhang contributed equally to this work.

\section{Acknowledgments}

The authors wish to thank all the clinical doctors who helped them in the Chinese PLA General Hospital. National Natural Science Foundation of China supports this research. The number is 81301564 . The funders had no role in study design, data collection and analysis, decision to publish, or preparation of the paper.

\section{References}

[1] Y. Assouline-Dayan, C. Chang, A. Greenspan, Y. Shoenfeld, and M. E. Gershwin, "Pathogenesis and natural history of osteonecrosis," Seminars in Arthritis and Rheumatism, vol. 32, no. 2, pp. 94-124, 2002.

[2] K. Ohzono, M. Saito, K. Takaoka et al., "Natural history of nontraumatic avascular necrosis of the femoral head," The Journal of Bone and Joint Surgery, vol. 73, no. 1, pp. 68-72, 1991.

[3] R. J. Patterson, W. H. Bickel, and D. C. Dahlin, "Idiopathic avascular necrosis of the head of the femur. A study of fifty-two cases," The Journal of Bone and Joint Surgery: American Volume, vol. 46, pp. 267-282, 1964.

[4] R. Merle D'Aubigne, M. Postel, A. Mazabraud, P. Massias, J. Gueguen, and P. France, "Idiopathic necrosis of the femoral head in adults," Journal of Bone and Joint Surgery-Series B, vol. 47, no. 4, pp. 612-633, 1965.

[5] H. Terayama, M. Ishikawa, Y. Yasunaga et al., "Prevention of osteonecrosis by intravenous administration of human peripheral blood-derived CD34-positive cells in a rat osteonecrosis model," Journal of Tissue Engineering and Regenerative Medicine, vol. 5, no. 1, pp. 32-40, 2011. 
[6] J. P. Jones Jr., "Risk factors potentially activating intravascular coagulation and causing nontraumatic osteonecrosis," in Osteonecrosis: Etiology, Diagnosis and Treatment, J. R. Urbaniac and J. P. Jones Jr., Eds., pp. 89-96, American Academy of Orthopaedic Surgeons, Rosemont, NJ, USA, 1997.

[7] M. G. Lykissas, I. D. Gelalis, I. P. Kostas-Agnantis, G. Vozonelos, and A. V. Korompilias, "The role of hypercoagulability in the development of osteonecrosis of the femoral head," Orthopedic Reviews, vol. 4, no. 2, article e17, 2012.

[8] C. Wermes, K.-W. Sykora, F. Bergmann, and B. Reller, "Severe protein $\mathrm{C}$ deficiency and aseptic osteonecrosis of the hip joint: a case report," European Journal of Pediatrics, vol. 158, no. 3, pp. S159-S161, 1999.

[9] C. G. Zalavras, G. Vartholomatos, E. Dokou, and K. N. Malizos, "Factor V Leiden and prothrombin gene mutations in femoral head osteonecrosis," Thrombosis \& Haemostasis, vol. 87, no. 6, pp. 1079-1080, 2002.

[10] A. Björkman, P. J. Svensson, A. Hillarp, I. M. Burtscher, A. Rünow, and G. Benoni, "Factor V Leiden and prothrombin gene mutation: risk factors for osteonecrosis of the femoral head in adults," Clinical Orthopaedics and Related Research, no. 425, pp. 168-172, 2004.

[11] S. A. Zimmerman and R. E. Ware, "Inherited DNA mutations contributing to thrombotic complications in patients with sickle cell disease," American Journal of Hematology, vol. 59, no. 4, pp. 267-272, 1998.

[12] L. A. J. Kluijtmans, L. P. W. J. van Den Heuvel, G. H. J. Boers et al., "Molecular genetic analysis in mild hyperhomocysteinemia: a common mutation in the methylenetetrahydrofolate reductase gene is a genetic risk factor for cardiovascular disease," The American Journal of Human Genetics, vol. 58, no. 1, pp. 35-41, 1996.

[13] D. Gemmati, M. L. Serino, C. Trivellato, S. Fiorini, and G. L. Scapoli, "C677T substitution in the methylenetetrahydrofolate reductase gene as a risk factor for venous thrombosis and arterial disease in selected patients," Haematologica, vol. 84, no. 9, pp. 824-828, 1999.

[14] C. J. Glueck, R. A. Freiberg, R. N. Fontaine, T. Tracy, and P. Wang, "Hypofibrinolysis, thrombophilia, osteonecrosis," Clinical Orthopaedics and Related Research, no. 386, pp. 19-33, 2001.

[15] S. Li, Z. W. Pan Wen, T. Guo, H. Mu, and F. Sun, "Association study of non-traumatic avascular necrosis of the femoral head in TCM," Meeting Minutes, pp. 78-82, 2013.

[16] A. Kutlar, F. Kutlar, I. Turker, and C. Tural, "The methylene tetrahydrofolate reductase (C677T) mutation as a potential risk factor for avascular necrosis in sickle cell disease," Hemoglobin, vol. 25, no. 2, pp. 213-217, 2001.

[17] B. Bernbeck, C. Mauz-Körholz, R. B. Zotz, and U. Göbel, "Methylenetetrahydrofolate reductase gene polymorphism and glucocorticoid intake in children with ALL and aseptic osteonecrosis," Klinische Padiatrie, vol. 215, no. 6, pp. 327-331, 2003.

[18] T. Asano, K. A. Takahashi, M. Fujioka et al., "Relationship between postrenal transplant osteonecrosis of the femoral head and gene polymorphisms related to the coagulation and fibrinolytic systems in Japanese subjects," Transplantation, vol. 77, no. 2, pp. 220-225, 2004.

[19] W. Cochran, "The combination of estimates from different experiments," Biometrics, vol. 10, no. 1, pp. 101-129, 1954.

[20] N. Mantel and W. Haenszel, "Statistical aspects of the analysis of data from retrospective studies of disease," Journal of the National Cancer Institute, vol. 22, no. 4, pp. 719-748, 1959.
[21] R. DerSimonian and N. Laird, "Meta-analysis in clinical trials," Controlled Clinical Trials, vol. 7, no. 3, pp. 177-188, 1986.

[22] M. Egger, G. D. Smith, M. Schneider, and C. Minder, "Bias in meta-analysis detected by a simple, graphical test," The British Medical Journal, vol. 315, no. 7109, pp. 629-634, 1997.

[23] C. J. Glueck, R. N. Fontaine, R. Gruppo et al., "The plasminogen activator inhibitor-1 gene, hypofibrinolysis, and osteonecrosis," Clinical Orthopaedics and Related Research, no. 366, pp. 133-146, 1999.

[24] C. G. Zalavras, K. N. Malizos, E. Dokou, and G. Vartholomatos, "The $677 \mathrm{C} \rightarrow \mathrm{T}$ mutation of the methylene-tetra-hydrofolate reductase gene in the pathogenesis of osteonecrosis of the femoral head," Haematologica, vol. 87, no. 1, pp. 111-112, 2002.

[25] A. Celik, D. Tekis, F. Saglam et al., "Association of corticosteroids and factor V, prothrombin, and MTHFR gene mutations with avascular osteonecrosis in renal allograft recipients," Transplantation Proceedings, vol. 38, no. 2, pp. 512-516, 2006.

[26] J.-D. Chang, M. Hur, S.-S. Lee, J.-H. Yoo, and K. M. Lee, "Genetic background of nontraumatic osteonecrosis of the femoral head in the Korean population," Clinical Orthopaedics and Related Research, vol. 466, no. 5, pp. 1041-1046, 2008.

[27] B. Liu, Z. Li, W. Sun, B. Wang, S. Shi, and H. Min, "Relationship between alcohol induced osteonecrosis of femoral head and single nucleotide polymorphisms of methylene tetrahydrofolate reductase 677 C/T," Zhongguo Xiu Fu Chong Jian Wai Ke Za Zhi, vol. 23, no. 9, pp. 1079-1082, 2009.

[28] T.-H. Kim, J. M. Hong, H.-J. Kim, E. K. Park, and S.-Y. Kim, "Lack of association of MTHFR gene polymorphisms with the risk of osteonecrosis of the femoral head in a Korean population," Molecules and Cells, vol. 29, no. 4, pp. 343-348, 2010.

[29] J. Gagala, M. Buraczynska, T. Mazurkiewicz, and A. Ksiazek, "Prevalence of genetic risk factors related with thrombophilia and hypofibrinolysis in patients with osteonecrosis of the femoral head in Poland," BMC Musculoskeletal Disorders, vol. 14, article 264, 2013.

[30] V. Ramensky, P. Bork, and S. Sunyaev, "Human nonsynonymous SNPs: server and survey," Nucleic Acids Research, vol. 30, no. 17, pp. 3894-3900, 2002.

[31] L. M. Dong, J. D. Potter, E. White, C. M. Ulrich, L. R. Cardon, and U. Peters, "Genetic susceptibility to cancer: the role of polymorphisms in candidate genes," The Journal of the American Medical Association, vol. 299, no. 20, pp. 2423-2436, 2008.

[32] D. Girelli, S. Friso, E. Trabetti et al., "Methylenetetrahydrofolate reductase C677T mutation, plasma homocysteine, and folate in subjects from northern Italy with or without angiographically documented severe coronary atherosclerotic disease: evidence for an important genetic-environmental interaction," Blood, vol. 91, no. 11, pp. 4158-4163, 1998.

[33] P. Frosst, H. J. Blom, R. Milos et al., "A candidate genetic risk factor for vascular disease: a common mutation in methylenetetrahydrofolate reductase," Nature Genetics, vol. 10, no. 1, pp. 111113, 1995.

[34] X.-F. Shang, H. Su, W.-W. Chang, C.-C. Wang, Q. Han, and Z.-W. $\mathrm{Xu}$, "Association between MTHFR C677T polymorphism and osteonecrosis of the femoral head: a meta-analysis," Molecular Biology Reports, vol. 39, no. 6, pp. 7089-7094, 2012. 


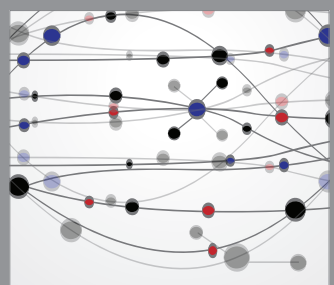

The Scientific World Journal
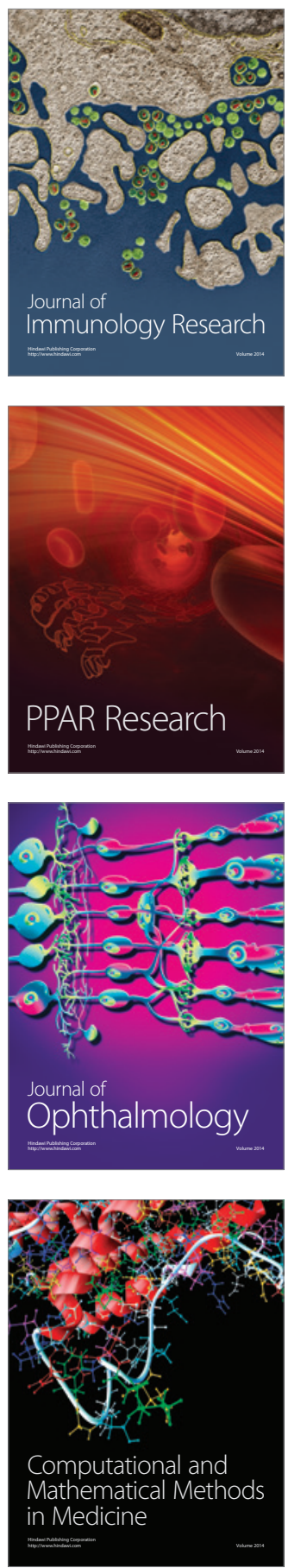

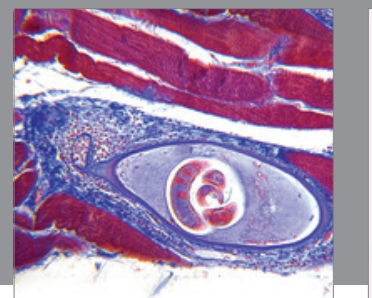

Gastroenterology

Research and Practice
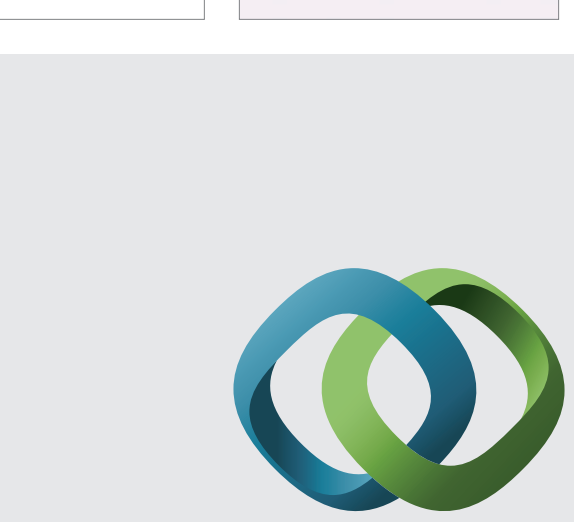

\section{Hindawi}

Submit your manuscripts at

http://www.hindawi.com
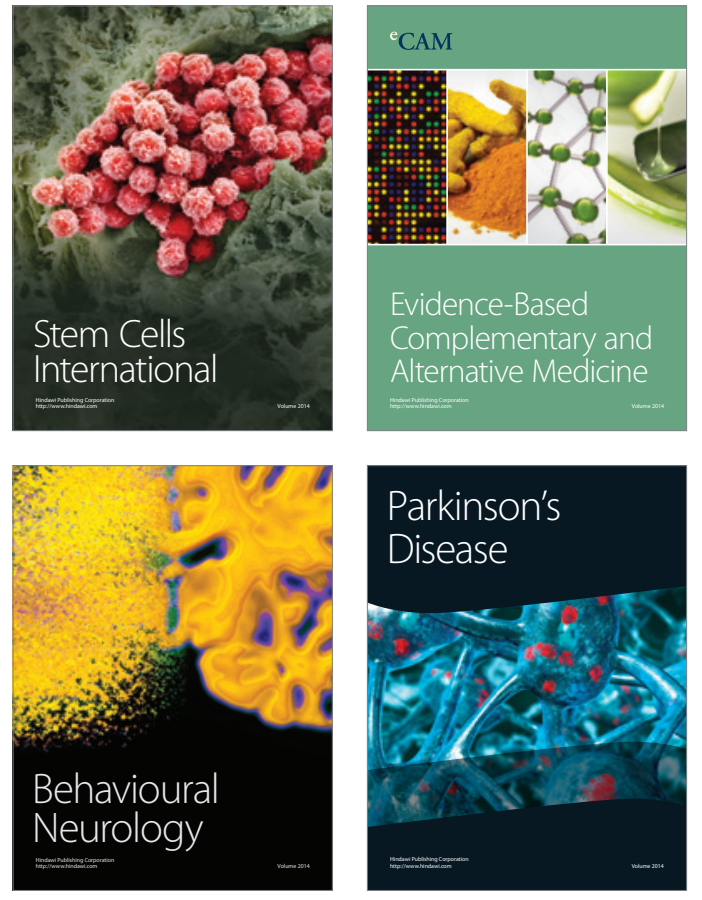
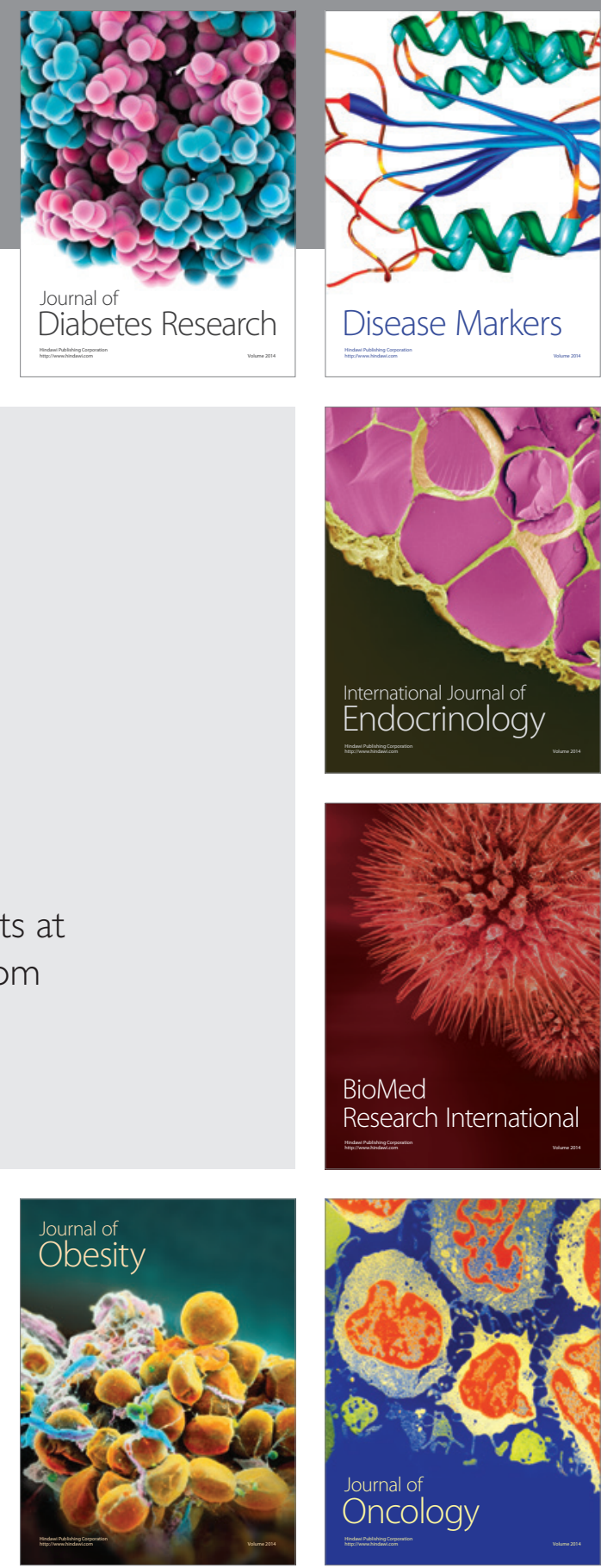

Disease Markers
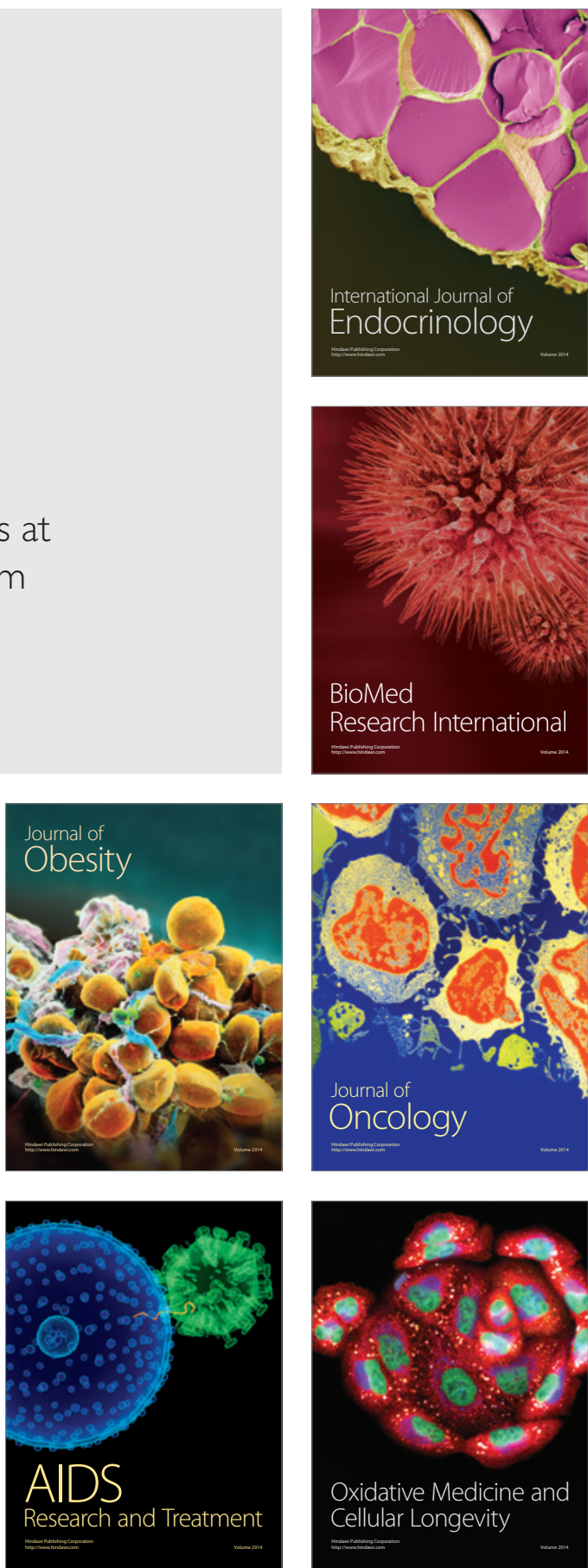